\title{
Impact of Topology on Energy Consumption in Wireless Sensor Networks
}

\author{
B. G. Awatef, N. Nejeh and K. Abdennaceur \\ National Engineering School of Sfax (ENIS), Sfax, Tunisia \\ Received 23 February 2014; Accepted 24 May 2014 \\ Publication 4 August 2014
}

\begin{abstract}
Energy consumption is often regarded as a fundamental parameter in the context of availability in the wireless sensor networks (WSNs). This parameter poses energy problems particularly if the application must function for a long time. For the WSNs, it is impossible to reload or replace the batteries of nodes after their exhaustion. Several factors can be a source of energy over-consumption: mobility, retransmissions, the node position (relay or gateway), ... The network topology can also damage the nodes batteries. Generally, the transmission in 1-hop increases the energy consumption if the distance is rather high. The transmission in K-hops solves the problem but weakened the energy of the intermediate nodes. In this paper, we will focus on the impact of topology on energy consumption and determine optimal topology maximizing lifetime of WSNs.
\end{abstract}

Keywords: wireless sensor networks (WSNs), clustering, clusterhead, energy, topology.

\section{Introduction}

A WSN represents a set of small devices called sensors deployed in a geographical zone. The sensor is able to supervise a phenomenon and to send information to one or more point of collection inter-connected via a wireless connectivity ([1], [2]). Figure 1 represents the general diagram of

Journal of Machine to Machine Communications, Vol. 1, 145-160.

doi: 10.13052/jmmc2246-137X.124

(c) 2014 River Publishers. All rights reserved. 
WSNs: each sensor takes a physical greatness related to a noticed event, treat and transfer this data to a base station which transmits it towards the administrator.

Energy consumption (network lifetime) presents the most important metric in the performance evaluation of a WSNs ([3], [4]). Indeed, the lifetime is regarded as a fundamental parameter in the context of availability in the WSNs [5]. Energy consumption depends on several factors: state of the radio operator module (emission, reception or snooze for 802.15.4), retransmissions, idle listening, control packets, overhearing, collisions, ... [6]. The network topology represents also a major factor on energy consumption, indeed, a transmission in 1-hop requires a higher power if the distance between the nodes is high. A transmission in K-hops consumes less energy (for the initial node), but, can damage the batteries of the intermediate nodes.

Several researchers choose the transmission K-hops ([1,7-11]) in order to minimize the transmission range. Others choose the transmission with 1-hop ([12-14]) without taking into account the distance between a source and a destination.

In this paper, we proposed an energy efficient clustering algorithm. A node with lower mobility, higher residual energy, higher degree (the number of nodes in its neighborhood of 1-hop) and closer to the base station is more likely elected as a clusterhead. Then, we want to determine optimal topology based on the clustering and minimizing energy consumption for the networks. Simulation results are described in the last section.

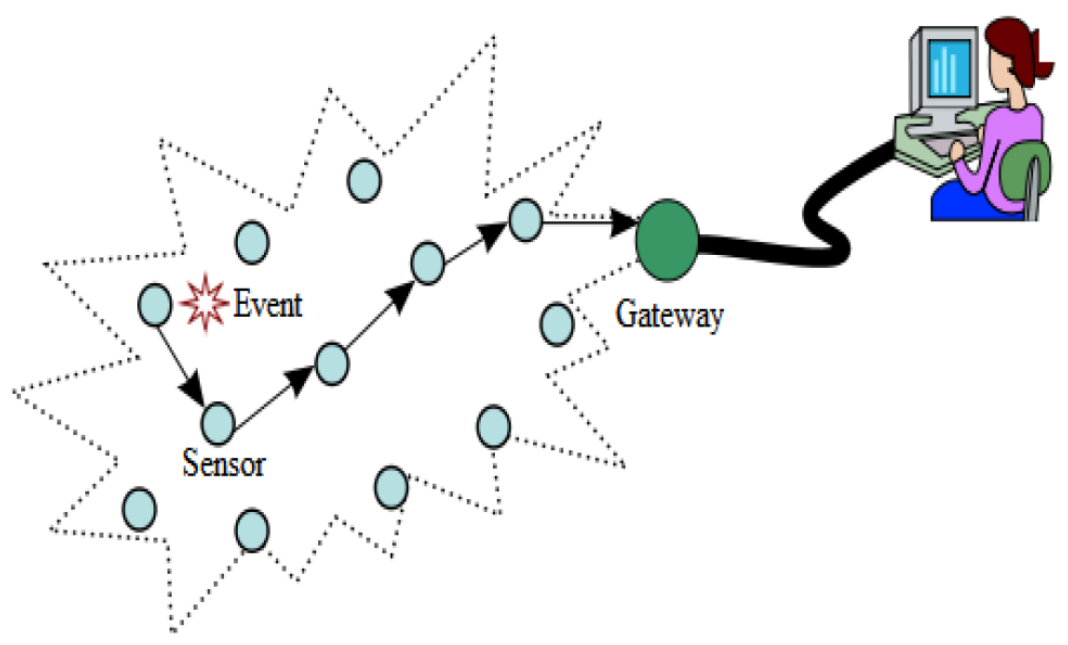

Figure 1 WSNs 


\section{Model and Notation}

For better understanding the operation of a true WSN and thereafter facilitating its design, its deployment and its analysis, we will propose a model as simple as possible.

We model the WSN by a graph $\mathrm{G}=(\mathrm{V}, \mathrm{E})$ where $\mathrm{V}$ is the whole of sensors and $\mathrm{E}=\{(\mathrm{u}, \mathrm{v}) \subset \mathrm{V} 2 \mid \mathrm{D}(\mathrm{u}, \mathrm{v}) \leq \mathrm{R}\}$ is the wireless connections between nodes. $\mathrm{R}$ is the communication range, and $\mathrm{D}(\mathrm{u}, \mathrm{v})$ defines the Euclidean distance (the distance is calculated by the power of transmission) between the nodes $\mathrm{u}$ and $\mathrm{v}$.

The properties are indicated in the Table 1.

\section{MEDD-BS Clustering Algorithms}

In this section, we propose a new clustering algorithm called MEDD-BS (Mobility Energy Degree Distance to Base Station) Clustering Algorithm for the wireless sensors networks of which the goal is the minimization of the energy consumption of WSNs.

\subsection{Mobility Model}

Mobility is the leading cause of topology changes in the sensors networks. It should be essential to integrate mobility metric for the clusterheads election and the clusters' formation.

There exist several applications where the nodes mobility is low like the mobility of a node deployed in the patient body to measure his physiological data. Other applications are characterized by a high nodes mobility, like the

Table 1 Parameters definition

\begin{tabular}{ll}
\hline $\mathbf{N}$ & Number of nodes. \\
ID $(\mathbf{u})$ & Identifier of node u \\
$\mathbf{D}(\mathbf{u})$ & Connectivity degree of u. \\
$\mathbf{M}(\mathbf{u})$ & Mobility of u. \\
Ec/com $(\mathbf{u})$ & Energy consumption by communication unit of u. \\
DisBS $(\mathbf{u})$ & Distance between the node u and the base station. \\
Neigh $(\mathbf{u})$ & Whole of nodes in the neighborhood of 1-hop of u. \\
$\mathbf{D}(\mathbf{u})$ & Degree of u. \\
Weight $(\mathbf{u})$ & Weight of u. \\
State (u) & State of u. : "CH" (clusterhead) and "Nm" (nodesmember) \\
T & Period of standby mode (deactivation period). \\
\hline
\end{tabular}


mobility of a node deployed in the player body who runs in order to measure his speed.

So, we will define three mobility levels for sensors:

- Level 1: nodes speed is very weak in this case, speed varies between 0 and $5 \mathrm{~km} / \mathrm{h}$.

- Level 2: nodes speed is average, in this case, speed varies between $5 \mathrm{~km} / \mathrm{h}$ and $20 \mathrm{~km} / \mathrm{h}$.

- Level 3: nodes speed is high, in this case, speed varies between $20 \mathrm{~km} / \mathrm{h}$ and $44 \mathrm{~km} / \mathrm{h}$.

We suppose that the sensors speed is constant. The sensor mobility is characterized by the mobility level and can have the following values:

- $\mathrm{M}(\mathrm{U})=1$, if the node speed $\mathrm{U}$ belongs to first level.

- $\mathrm{M}(\mathrm{U})=2$, if the node speed $\mathrm{U}$ belongs to second level.

- $\mathrm{M}(\mathrm{U})=3$, if the node speed $\mathrm{U}$ belongs to third level.

We also suppose that sensors nodes know in advance their mobility levels and that the nodes having meant and high mobility will not take part in the clusterheads election phase. The purpose of this assumption is to maintain the stability of the structure. We are interested in this paper by the nodes deployed in the human bodies, for that, the consumed power by the mobility of nodes is not considered into account.

\subsection{Energy Consumption Model}

The energy consumption rate in the sensors networks represents the most important metric in the performances "evaluation phase. This parameter depends on the used nodes" characteristics (standby mode, nature of data processing, transmitted power, ...), and nodes behavior during the communication (retransmission, congestion, diffusion of the messages, ... ) [15].

The consumed power by sensor is that the consumed powers by these capture units, treatment units and communication units. So the energy consumption formula is defined as follows [16]:

$$
E c=E c / \text { capture }+E c / \text { treatement }+ \text { Ec/communication }
$$

Where:

- Ec/capture: is the energy consumed by a sensor during the capture unit activation. This energy depends primarily on the type of detected event (image, its, temperature...) and of the tasks to be realized by this unit (sampling, conversion....). 
- Ec/treatment: is the energy consumed by the sensor during the activation of its treatment unit.

- Ec/communication: is the energy consumed by the sensor during the activation of its communication unit.

Generally, the consumed energy by sensors during communication is larger than those consumed by the treatment unit and the capture unit. Indeed, the transmission of a bit of information can consume as much as the execution of a few thousands instructions [17]. For that, we can neglect the energy of the capture unit, and the treatment unit compared to the energy consumed by the communication unit. In this case, the Equation (1) will be:

$$
E c=E c / \text { communication }
$$

The communication energy breaks up into emission energy and reception energy:

$$
E c=E_{T X}+E_{R X}
$$

Referring to [18], the transmission energy and reception energy are defined as follows:

$$
\begin{gathered}
E_{T X}=E_{\text {elec }} \times K+\varepsilon_{\text {amp }} \times K \times d^{\lambda} \\
E_{R X}=E_{\text {elec }} \times K
\end{gathered}
$$

Where:

- K: message length (bits).

- D: distance between transmitting node and receiving node (m).

- $\lambda$ : of way loss exhibitor, $\lambda=2$.

- Eelec: emission / reception energy, Eelec $=50 \mathrm{~nJ} / \mathrm{bit}$.

- $\epsilon$ amp: transmission amplification coefficient, $\epsilon \mathrm{amp}=100 \mathrm{pJ} / \mathrm{bit} / \mathrm{m}^{2}$

In [13], the authors compared the consumed power by a clusterhead by carrying out the aggregation of received messages with that consumed without aggregation. They showed that when the energy considered for aggregation is lower than a limit value $(1 \mu \mathrm{J} / \mathrm{bit} / \mathrm{signal})$, then, the transmission with aggregation requires a weaker energy than that without aggregation.

We suppose that the aggregation energy cost respects the limit value introduced into [13]. The power consumed by a clusterhead during the transmission towards the base station will be: 


$$
E_{T X}(c h)=E_{D A} \times K+E_{T X}(K, d)
$$

Where $\mathrm{E}_{\mathrm{DA}}$ : power consumed during aggregation.

\subsection{Clusterheads Election Procedure}

Step 1: Each node sends a message "hello" for the discovery of 1-hop neighborhood.

Step 2: Nodes having a low level of mobility $(M(u)=1)$ calculate their weights, the weight is calculated as follows:

$$
\text { Weight }(u)=E c(u)+\frac{1}{D(u)}+\frac{\operatorname{DisBS}(u)}{200}
$$

We devide DisBS by 200 in order to take into account three parameters (three parameters will be between 0 and 1).

Step 3: The nodes diffuse their weights towards their neighbors (1-hop).

Step 4: The node which has the weakest weight is declared like clusterhead by putting its state $=$ " $\mathrm{CH}$ " and sends a message "clusterhead_elected" (containing its identity) to its neighbors.

Step 5: The neighbors receiving this message, declare themselves like "Nm", send to the clusterhead a message "clusterhead_accepted", and record the identity of their clusterheads in their databases.

\subsection{Clusterheads Change Procedure}

This procedure is started if the waste energy of each $\mathrm{CH}$ will be lower than $40 \%$ of its initial energy.

Step 1: The clusterhead sends to its neighbors (1-hop) a message "clusterhead-changes", and is declared like "Nm".

Step 2: The nodes having a low mobility calculate and send their weights.

Step 3: The node having the weakest weight is declared like " $\mathrm{CH}$ ", and diffuses a message "clusterhead_elected". 
Step 4: The neighbors send to the clusterhead a message "clusterhead_accepted", and record the identity of their clusterhead in their databases.

\subsection{Organigram}

The organigram of MEDD-BS algorithm is the following:

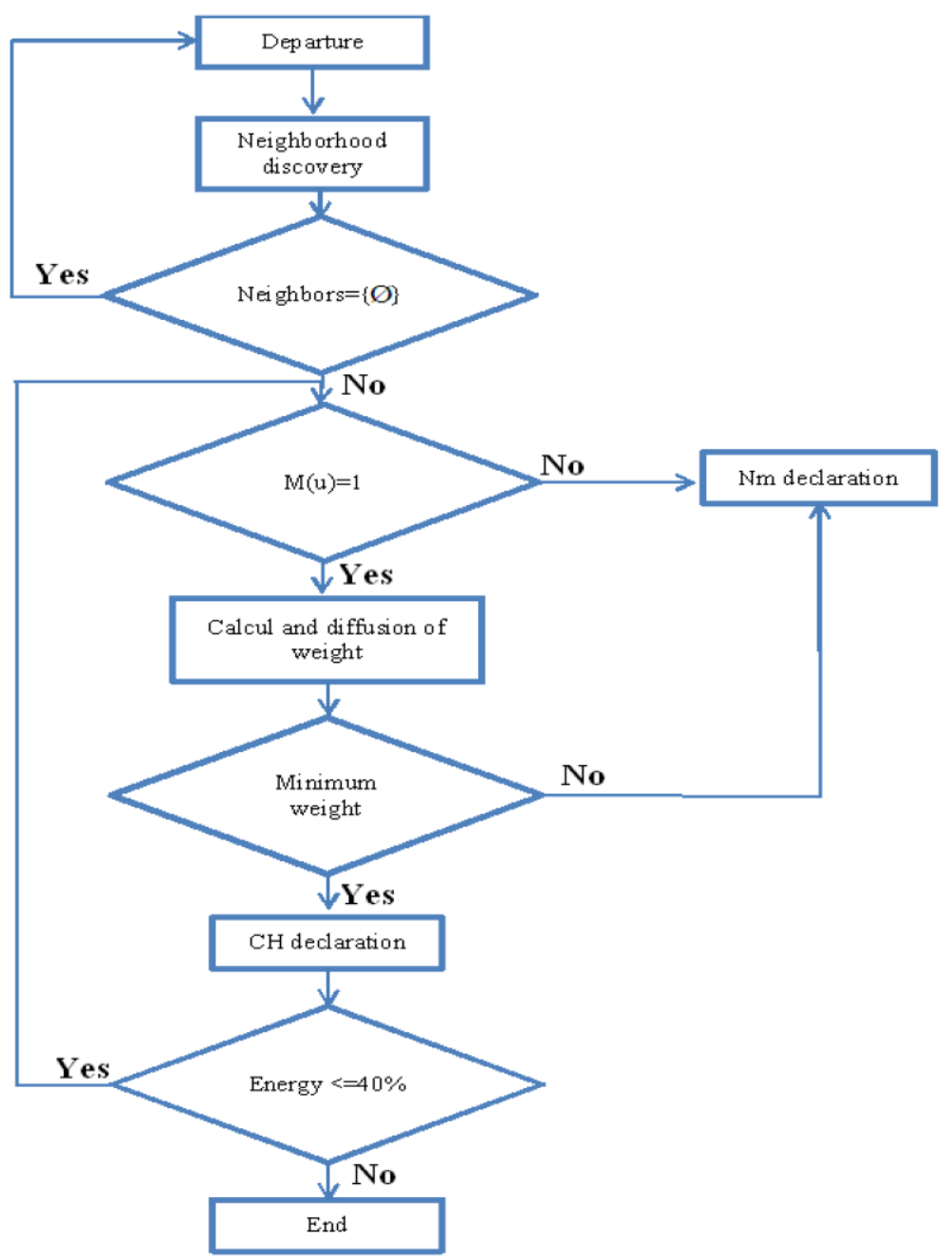

Figure 2 Organigram 


\section{Clusters Topology 1-HOP and K-HOPS}

In the intra-cluster transmission, any member can be either 1-hop or k-hops of its cluster-head (Figure 3). In the cluster with 1-hop, the cluster-head is directly connected to any node member. In a cluster with K-hops, it is essential to define a procedure which calculates the way between node and its cluster-head.

The information routing towards the base station can be done into 1-hop (Figure 4) or in K-hops (Figure 5).

Thus, it is possible for a clustering strategy to define four topologies (Table 2).

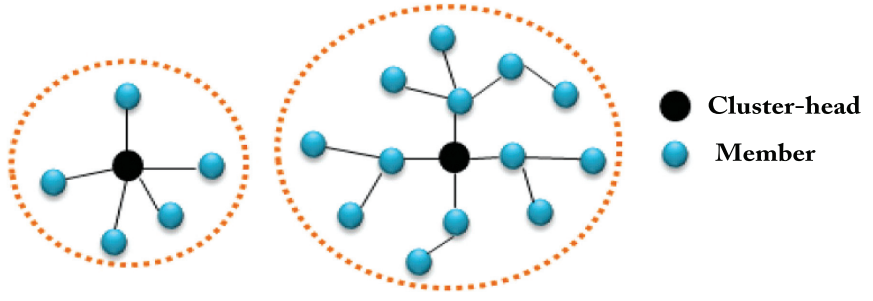

Figure 3 Connectivity intra-cluster

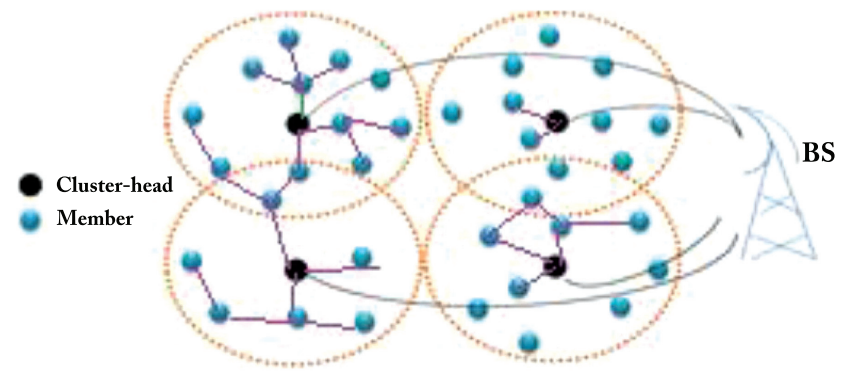

Figure 4 Connectivity inter-cluster 1-hop

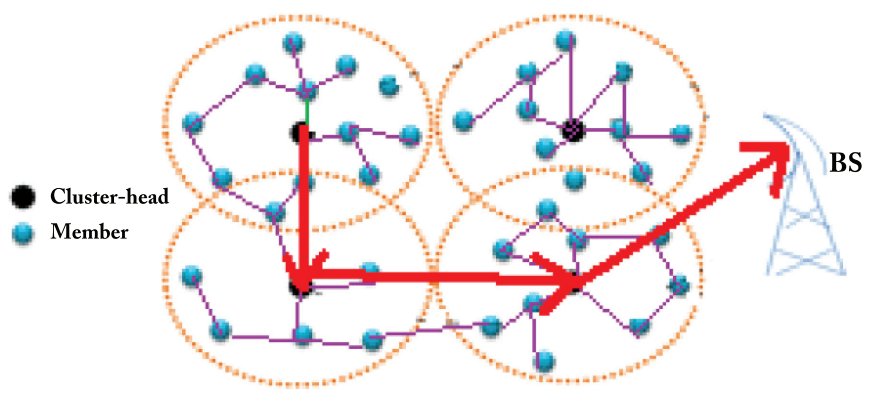

Figure 5 Connectivity inter-cluster k-hop 
Table 2 Topologies

\begin{tabular}{lll}
\hline & 1-hop & K-hop \\
& Inter-cluster & Inter-cluster \\
\hline 1- hop & Intra-cluster : 1-hop & Intra-cluster : 1-hop \\
Intra-cluster & Inter-cluster : 1-hop & Inter-cluster : k-hops \\
K-hops & Intra-cluster : k-hops & Intra-cluster : k-hops \\
Intra-cluster & Inter-cluster : 1-hop & Inter-cluster : k-hops \\
\hline
\end{tabular}

\section{Determination of the Optimal Topology}

We wish thereafter to determine the topology optimizing energy consumption. For that, we will compare the power consumption in only one hop (E1) between a source $\mathrm{S}$ and a destination $\mathrm{D}$ and that consumed in two hops (E2).

Power consumption to directly transmit a message from $\mathrm{S}$ to $\mathrm{D}$ is equal to a transmission energy consumed by $S$ and a reception energy consumed by $\mathrm{D}$ :

$$
E 1=E_{\text {elec }} \times K+\varepsilon_{\text {amp }} \times K \times d 3^{\lambda}+E_{\text {elec }} \times K
$$

The Equation (8) becomes after simplification:

$$
E 1=2 \times E_{\text {elec }} \times K+\varepsilon_{\text {amp }} \times K \times d 3^{\lambda}
$$

Power consumption to transmit a message from $\mathrm{S}$ to $\mathrm{D}$ by $\mathrm{G}$ is equal to an energy of transmission consumed by $\mathrm{S}$, an energy of reception consumed by $\mathrm{G}$, an energy of transmission consumed by $\mathrm{G}$ and an energy of reception consumed by D:

$E 2=E_{\text {elec }} \times K+\varepsilon_{a m p} \times K \times d 1^{\lambda}+E_{\text {elc }} \times K+\varepsilon_{\text {amp }} \times K \times d 2^{\lambda}+E_{\text {elec }} \times K$

The Equation (10) becomes after simplification:

$$
E 2=4 \times E_{\text {elec }} \times K+\varepsilon_{\text {amp }} \times K \times\left(d 1^{\lambda}+d 2^{\lambda}\right)
$$

We want now to compare two energies E1 and E2, then, we will calculate E2-E1:

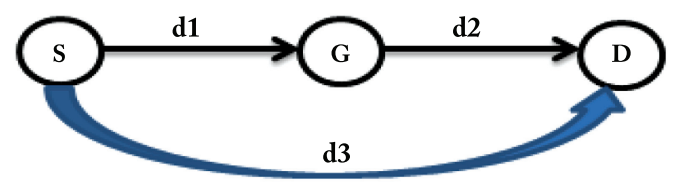

Figure 6 Transmission 1-hop and 2-hops 


$$
E 2-E 1=2 \times E_{\text {elec }} \times K-\varepsilon_{a m p} \times K \times 2 \times d 1 \times d 2
$$

If E2-E1 is positive, then, the transmission in 1-hop is more profitable:

If:

$$
E 2-E \geq 0
$$

Then:

$$
2 \times E_{\text {elec }} \times K-\varepsilon_{\text {amp }} \times K \times 2 \times d 1 \times d 2 \geq 0
$$

After simplification, we will have:

$$
d 1 \times d 2 \leq \frac{E_{e l c}}{\epsilon_{a m p}}
$$

Then:

$$
d 1 \times d 2 \leq 500 m^{2}
$$

So, the transmission in 1-hop is profitable when $\mathrm{d} 1 * \mathrm{~d} 2 \leq 500$ and the transmission in 2-hops is profitable when $\mathrm{d} 1 * \mathrm{~d} 2 \geq 500 \mathrm{~m}^{2}$.

Within a cluster (transmission intra-cluster), the distances between the nodes are generally weak (about $10 \mathrm{~m}$ and $20 \mathrm{~m}$ ), then, the multiplication between two distances is lower than 500, which favour the transmission in 1-hop.

For the inter-cluster transmission, the distances between nodes are generally larger (about $70 \mathrm{~m}$ and $100 \mathrm{~m}$ ), then, the multiplication between two distances is higher than 500, which favour the transmission into 2-hop.

So, we can conclude that the optimal topology is topology $1 \mathrm{k}$ (1-hop intra-cluster and K-hops inter-cluster).

\section{Simulation Results}

The simulation results were implemented using Matlab 7.0.1 tool. The network of first level is composed of set of sensors. The number of nodes in the sensor network varies between 10 and 200 nodes. The mobility of each sensor is supposed constant, and a speed is dedicated for each level of mobility: level 1: $1 \mathrm{~km} / \mathrm{h}$, level 2: $5 \mathrm{~km} / \mathrm{h}$ and level $3: 20 \mathrm{~km} / \mathrm{h}$. The initial energy for each sensor is equal to $0.5 \mathrm{~J}$. 
The simulation of the proposed algorithm was carried out during 10 intervals $\mathrm{T}$ (standby mode) in a space of $150 \mathrm{~m} \times 150 \mathrm{~m}$ and the range of the nodes (Tx-Arranges) is $40 \mathrm{~m}$. The size of a measured data package for sensors and envoy towards their clusterheads is 4000 bits. Figure 7 shows the communication structure of network with 30 nodes. In this figure, red o represent the cluster-head, yellow triangle represent the ordinary sensor node, blue lines represent the communication between cluster-head and ordinary sensor nodes, and black * represent the base station.

In this section, we will represent the results of our algorithm by varying the transmission topology. Figure 8 improves the results shows in the preceding

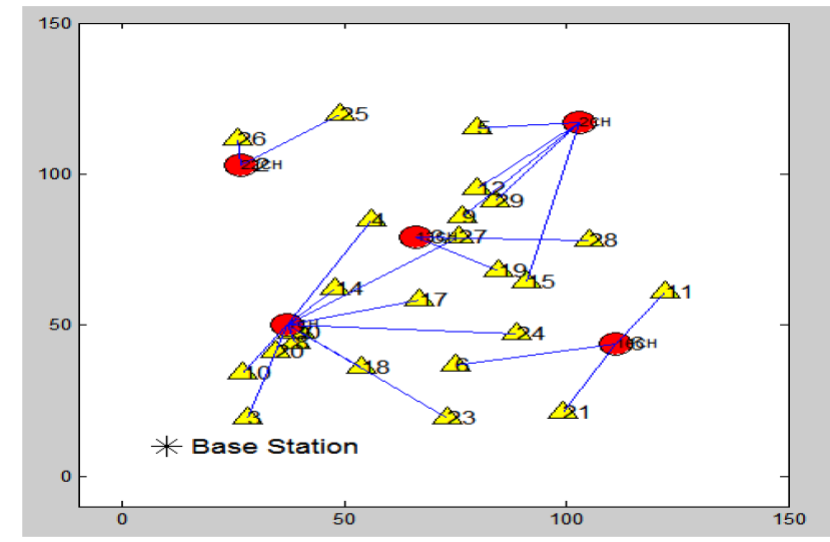

Figure 7 Communication structure

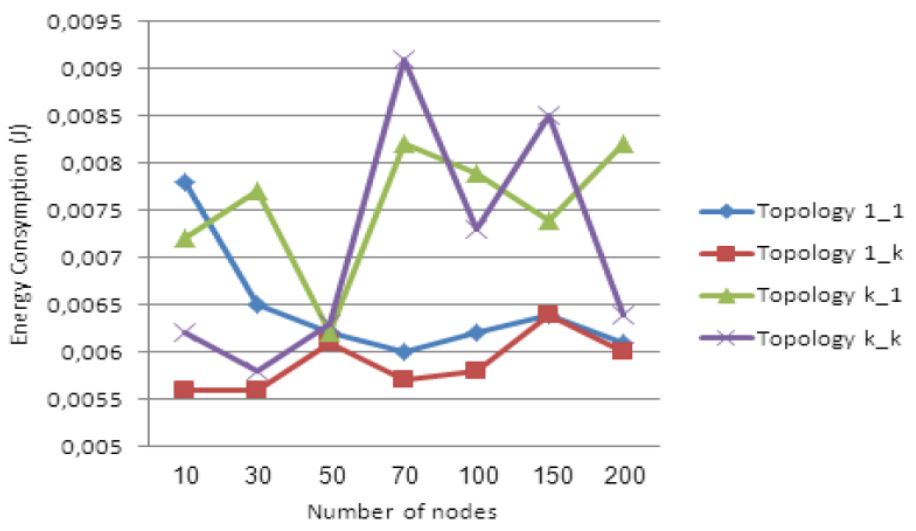

Figure 8 Energy consumption 
part. Topology $1-\mathrm{k}$ is topology which minimizes energy consumption for WSNs.

Figure 9 represents the medium number of constructed CHs VS number of nodes. We can notice that four topology give comparable values of $\mathrm{CHs}$. The transmissions 1-hop between members and its $\mathrm{CHs}$ increases the number of the built clusters. The transmissions k-hops increases the number of nodes in the cluster and, by consequence, the number of constructed cluster decrease.

Figure 10 represents the average number of transmitted packets to $\mathrm{CHs}$. "Topology 1 k" and "Topology k k" gives the highest values. For "Topology

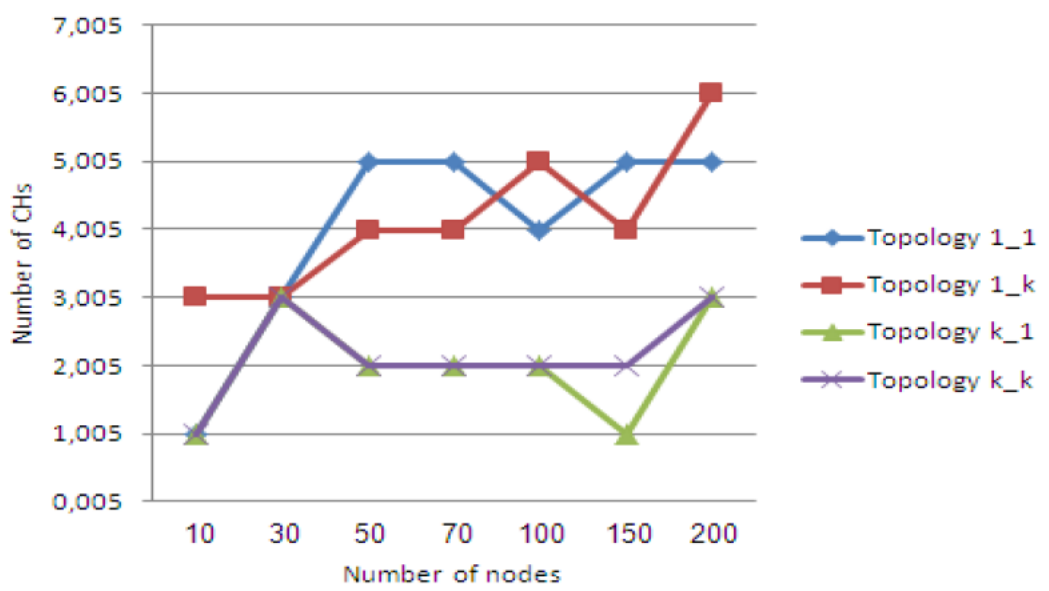

Figure 9 Number of $\mathrm{CHs}$

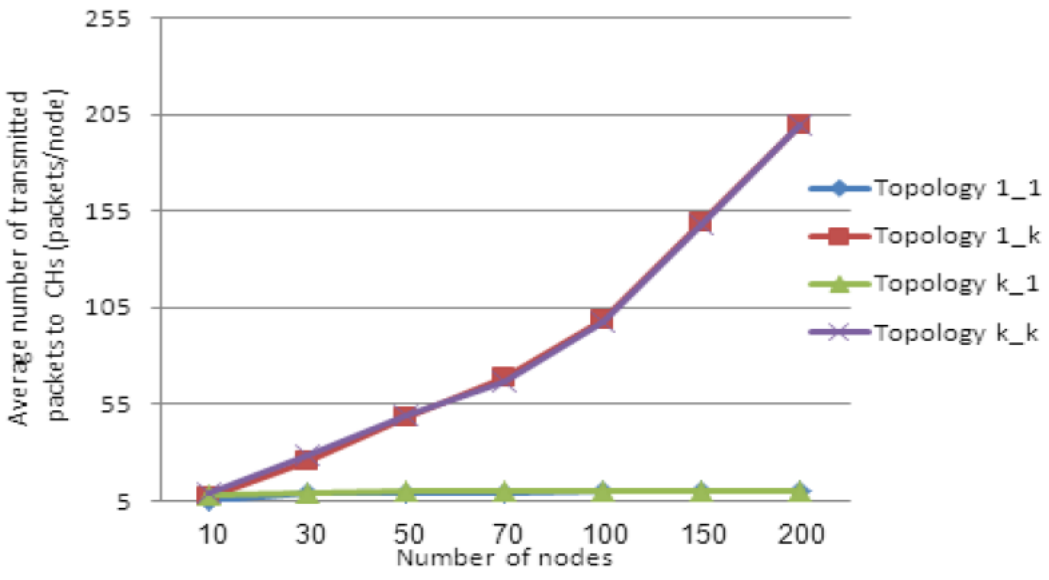

Figure 10 Transmitted packets to $\mathrm{CHs}$ 


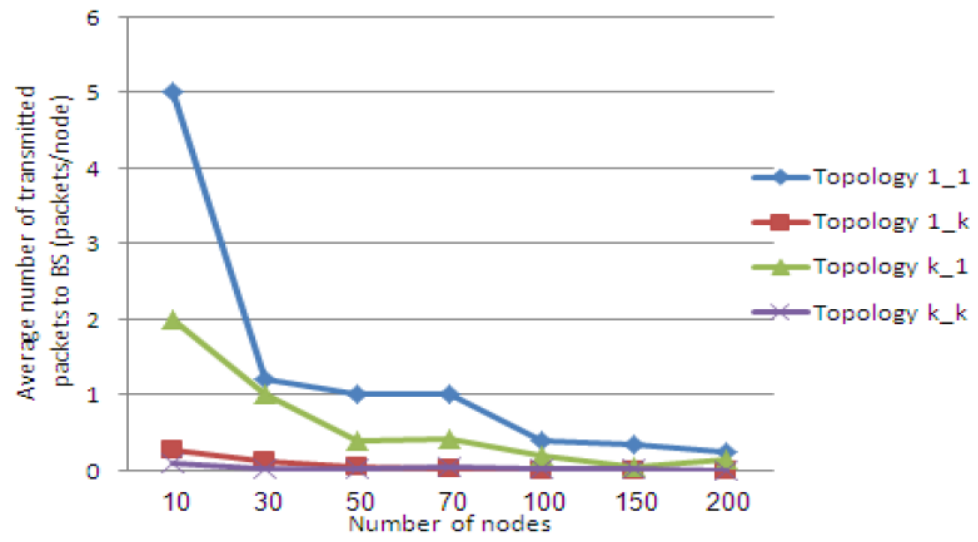

Figure 11 Transmitted packets to BS

1_k", a large number of $\mathrm{CHs}$ is remarked what makes the packages value higher. For "Topology k_k", a way multi-hops is carried out at the time of transmissions towards the BS, thus more number of packages are sent between CHs.

Figure 11 represents average number of transmitted packets to the BS. The lowest values are those of "Topology 1_k" and "Topology k_k", only one $\mathrm{CH}$ carries out the procedure of transmission towards the base station.

\section{Conclusion}

In this paper, we focussed on the impact of the topology on the energy consumption in the WSNs. Theoretical and simulation results have shown that the topology $1 \_\mathrm{k}$ (1-hop intra-cluster and k-hop inter-cluster) optimize energy consumption and increases by consequence the network lifetime. The next step should be the construction of a topology $1 \_\mathrm{K}$ which minimize the energy consumption of each node in the WSN.

\section{References}

[1] C. Tidjane Kone, "Conception de l"architecture d"un réseau de capteurs sans fil de grande dimension", PHD, University of Henri Poincaré Nancy I, October 2011.

[2] G. Chalhoub, "Les réseaux de capteurs sans fil", Ph.D, University of Clermont, Auvergne, 2010. 
[3] L. Samper, "Modélisations et Analyses de Réseaux de Capteurs", PHD, VERIMAG laboratory, April 2008.

[4] R. Kacimi, "Techniques de conservation d"énergie pour les réseaux de capteurs sans fil”, PHD, TOULOUSE UNIVERSITY, September 2009.

[5] M. Khan and J. Misic. "On the lifetime of wireless sensor networks", ACM Transactions on Sensor Networks (TOSN), Vol. 5, No. 5, 2009.

[6] R. Kuntz, "Medium access control facing the dynamics of wireless sensor networks", PHD, University of Strasbourg, 2010.

[7] D. Kumar, D. Trilok, C. Aseri and R. B. Patel, "EEHC: Energy efficient heterogeneous clustered scheme for wireless sensor networks", Computer Communication., Volume 32, Issue 4, pp 662-667, 4 March 2009.

[8] W. B. Heinzelman, "Application-Specific Protocol Architectures for Wireless Networks”, PHD, B.S.Cornell University, June 2000.

[9] Y. Ossama and S. Fahmy, "HEED: A hybrid energy-efficient distributed clustering approach for ad hoc sensor networks", IEEE Transactions on Mobile Computing, Volume 3, Issue 4, pp 366 - 379, 2004.

[10] Duan, Changmin et Hong Fan, "A distributed energy balance clustering protocol for heterogeneous wireless sensor neworks", In: Proc. Int. Conf. Wireless Communications, Networking and Mobile Computing WiCom, pp. 2469-2473, 2007.

[11] Zhenhua Yu, YuLiu and Yuanli Cai, "Design of an Energy-Efficient Distributed Multi-level Clustering Algorithm for Wireless Sensor Networks", Proc IEEE $4^{\text {th }}$ International Conference: Wireless Communications, Networking and Mobile Computing (WiCOM“08),2008.

[12] T. Neeta, G. Elangovan, S. Iyengar and N. Balakrishnan, "A message-efficient, distributed clustering algorithm for wireless sensor and actor networks", IEEE Int Multisensor Fusion and Integration for Intelligent Systems Conf, 2006.

[13] Wendi Rabiner Heinzelman, Anantha Chandrakasan, and Hari Balakrishnan, "Energy-Efficient Communication Protocol for Wireless Microsensor Networks", In : Proc IEEE 33rd Hawaii International Conference on System Sciences, 2000.

[14] CP. Low, C. Ping, C. Fang, J. Mee and Y. Hock Ang, "Load-balanced clustering algorithms for wireless sensor networks", Proc. IEEE Int Conf Communications ICC, 2007.

[15] V. Raghunathan, C. Schurgers, S. Park and M.B. Srivastava, "Energyaware wireless micro-sensor networks", IEEE Signal Processing Magazine, Vol. 19, No. 2, pp 40-50, 2002. 
[16] Mitton, Nathalie, Busson, Anthony et Fleury, Eric. "Self-organization in large scale adhoc networks", In : Mediterranean ad hoc Networking Workshop (Med-Hoc- Net“"04). Bodrum, Turquie, 2004.

[17] G. J. Pottie and W. J. Kaiser, "Wireless integrated network sensors", Commun. ACM, Volume 43 Issue 5, pp 51-58, May 2000.

[18] W. N. Richard and A. Boukerche, "Mobile data collector strategy for delay - sensitive applications over wireless sensor networks," Computer Communications, Volume 31, Issue 5, pp 1028-1039, 25 March 2008.

\section{Biographies}

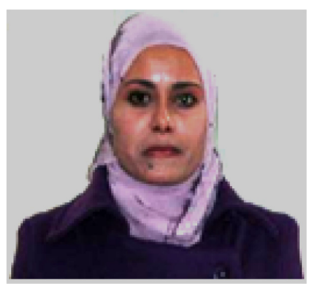

Awatef BENFRADJ was born in Gabes, Tunisia in 1983. She received the engineering degree in Telecommunications and Networks from the University of Gabes, in 2007. In 2009, she obtained the master degree in Networks from Higher School of Communication of Tunisia. Since 2011, she has been a researcher within the laboratory of electronics and information technology, Sfax University.

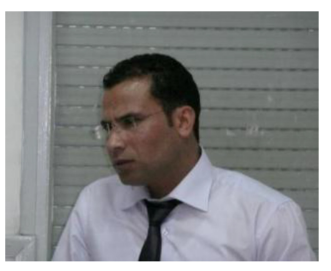

Nejah NASRI was born in Menzel Bouzaienne Village, in 1981. He received the B.S. and M.S. degrees in electronic engineering from the University of Sfax, in 2006 and the Ph.D. degree in electronic and telecommunication engineering from Toulouse University, France, in 2010. From 2006 to 2009, he was a Research Assistant with Higher Institute of Computer and Communication 
Techniques (ISITCom), Hammam Sousse, Tunisia. Since 2010, he has been an Assistant Professor with the Informatics Engineering Department, Gafsa University. He is the author of more than 70 articles. His research interests include engineering of wireless sensors networks and wireless underwater communication.

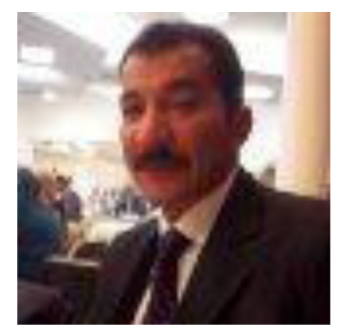

Abdennaceur KACHOURI was born in Sfax, Tunisia, in 1954. He received the engineering diploma from National school of Engineering of Sfax in 1981, a Master degree in Measurement and Instrumentation from National school of Bordeaux (ENSERB) of France in 1981, a Doctorate in Measurement and Instrumentation from ENSERB, in 1983. He "works" on several cooperation with communication research groups in Tunisia and France. Currently, he is Permanent Professor at ENIS School of Engineering and member in the "LETI" Laboratory ENIS Sfax. 